\title{
A STUDY ON CLINICAL EVALUATION, MRI \& ARTHROSCOPY IN CRUCIATE LIGAMENT \& MENISCAL INJURIES
}

Ashwin Kasturi1 ${ }^{1}$ E.Veeraji ${ }^{2}$, B.Arvind ${ }^{3}$, Ritesh Jaiswal ${ }^{4}$.

1. Assistant Professor, Department of Orthopedics, Malla Reddy Institute of Medical Sciences.

2. Senior Resident, Department of Orthopedics, Malla Reddy Institute of Medical Sciences.

3. Assistant Professor, Department of Orthopedics, Malla Reddy Institute of Medical Sciences.

4. Senior Resident, Department of Orthopedics, Malla Reddy Institute of Medical Sciences.

\section{CORRESPONDING AUTHOR}

Dr. Ashwin Kasturi,

Plot No- 524,

II Floor, Bagath Singh Nagar,

Beside JNTU, KPMB colony.

Email- ashwinkasturi@gmail.com

\section{HOW TO CITE THIS ARTICLE:}

Ashwin Kasturi, E. Veeraji, B. Arvind, Ritesh Jaiswal. "A Study on Clinical Evaluation, MRI \& Arthroscopy in Cruciate Ligament \& Meniscal Injuries". Journal of Evolution of Medical and Dental Sciences 2013; Vol2, Issue 25, June 24; Page: 4536-4541.

ABSTRACT: BACKGROUND: This study is to determine the importance of Clinical Examination, MRI and Arthroscopy findings of one over the other respectively, in assessing meniscal \& ligament injury of knee joint.

Knee joint is one of the most commonly injured joints because of its anatomical structure, its exposures to external forces and the functional demands placed on it. In the last four decades advances in understanding and treatment of knee instability have progressed at a rapid rate. Historically several investigations have shown that clinical diagnosis is approximately $70 \%$ accurate in diagnosing knee pathology.

STUDY DESIGN: This is an objective study in which, patients with complaints of pain and instability of knee attending to the Department of Orthopaedics, Malla Reddy Hospital (MRH) attached to Malla Reddy Institute of Medical Sciences (MRIMS), Surarum, Hyderabad, were studied. More than 200 patients who have reported to the outpatient department between $1^{\text {st }}$ January 2012 and $31^{\text {st }}$ August 2012, of which 50 patients underwent clinical examination, MRI and Arthroscopic evaluation.

RESULTS: All the 10 cases of isolated ACL injury and one case of only PCL injury, one case of both ACL \& PCL injury, there was equal correlation between Clinical, MRI \& Arthroscopy. Two PCL and bicruciate ligaments were diagnosed clinically, MRI and Arthroscopy. In both meniscal and cruciate ligament injuries 32 cases were diagnosed by clinical examination, out of which Arthroscopically 26 were diagnosed and 28 were diagnosed by MRI. In isolated meniscal injured there were five diagnosed on arthroscopy, eight on clinical examination \& four on MRI. Five were misdiagnosed clinically but on arthroscopy had no tear.

CONCLUSION: From our study we concluded that, for isolated ACL and PCL injuries Clinical, MRI and Arthroscopy are almost equal in diagnosing the condition. For Meniscal injuries the sensitivity was 


\section{ORIGINAL ARTICLE}

$88 \%$ and specificity was $97 \%$ by clinical examination, sensitivity was $93.5 \%$, specificity was $93 \%$ by MRI, sensitivity was $100 \%$ and specificity was $100 \%$ for Arthroscopy. In both Meniscal and Cruciate Ligament specificity was $100 \%$ and sensitivity was $90 \%$ by clinical examination, specificity was $98 \%$ and sensitivity was $93 \%$ for MRI, sensitivity was 100\% and specificity was 100\% for Arthroscopy. Therefore Arthroscopy is best indicator of Cruciate Ligament as well as Meniscal injuries.

KEYS WORDS: Menisci, ACL (Anterior Cruciate Ligament), PCL (Posterior Cruciate Ligament), Arthroscopy, MRI (Magnetic Resonance Imaging)

INTRODUCTION: In the last four decades advances in understanding and treatment of knee instability have progressed at a rapid rate. Refinements in physical examination techniques, improved radiographic modalities, advances in rehabilitation and improved surgical techniques have contributed to an improved ability to care patients with anterior cruciate ligament (ACL) deficient knee or knee with more complex instability pattern.

Knee joint is one of the most commonly injured joints because of its anatomical structure, its exposures to external forces and the functional demands placed on it. The term "IDK" (Internal Derangement of Knee) was originally coined by William Hey ${ }^{1}$. It is broadly used to describe the abnormalities of knee functions due to any cause but mostly traumatic.

The IDK includes tear of cruciate ligament and those of menisci. Anatomically no joint include as many intra-articular structures as knee joint does. They are strong but not to the extent so as to sustain violent rotation and sweep stresses at the knees ${ }^{2}$. Stability of knee is mainly controlled by these static structures apart from the muscle groups around the knees 3 . Hence in any injury to the knee joint it is imperative to assess the integrity of these internal structures.

Historically several investigations have shown that clinical diagnosis is approximately $70 \%$ accurate in diagnosing knee pathology $4,5,6$. The structure i.e., deranged following twisting injury requires knee sense of clinical judgment, radio graphics, MRI and at timed arthroscopy.

The assessment of ACL deficient knee has been considered by numerous authors over the past decade. Several new clinical tests to improve diagnosis of sagittal instability have been developed and attempts have been made to measure the displacement ${ }^{7}$. In 1980's several ligament testing devices were developed in an attempt to quantize anterior posterior displacement of knee joint. The Lachaman's, Pivot shift \& anterior drawer tests often vary from examiner, thus making comparisons difficult. Objective, quantitative testing devices provide opportunity to compare populations of patients more accurately.

Several investigations like x-ray, MRI and diagnostic arthroscopies have been considered. Simple x-rays have not benefited in assessing ligament laxity. MRI, though best non invasive modality of investigations but MRI is considered to be sensitive to meniscal and less sensitive to cruciate ligament injuries ${ }^{8,9}$.MR imaging can separate surgically significant from non significant meniscal lesions and is useful in the non invasive preoperative screening of suspected meniscal tear ${ }^{10}$.

Definite patterns in the location of intra meniscal signal that comes in contact with the meniscal surface. These patterns vary in the frequency of associated meniscal tears. Although menisci with internal signal in contact with the surface are usually torn, a tear is less likely if such signal is present on only one image. Tears may be identifiable on only one image plane ${ }^{11 .}$ 


\section{ORIGINAL ARTICLE}

MR imaging is useful in the preoperative evaluation of suspected meniscal tears. A majority of the false positive MR images involved the posterior horns of the menisci12. Unavoidable false positives may be related to confusion between fraying \& tear even for experienced radiologists ${ }^{13}$.Prospective interpretation of MR images of the lateral meniscus had a sensitivity of $82 \%$, specificity of $98 \%$, and accuracy of $93 \%$. For images of the medial meniscus, sensitivity of $82 \%$, specificity of $98 \%$, and accuracy of $93 \%{ }^{14}$.

Carefully performed clinical examination can give equal or better diagnosis of meniscal and ACL injuries in comparison to MRI scan. MRI may be used to rule out such injuries rather than to diagnose them ${ }^{15}$.

Clinical examination was accurate in $96.9 \%$ and MRI in $85.9 \%$ of medial meniscal injuries. For lateral meniscus injuries, clinical accuracy was $85.4 \%$ and MRI $73.8 \%$, MRI findings showed the lowest correlation with arthroscopic findings in lateral meniscus injuries. Clinical diagnostic performance was poorest in case of combined cruciate ligament and meniscal injuries ${ }^{16}$.

AIMS AND OBJECTIVES: To assess the importance of Clinical Examination, MRI and Arthroscopy findings one over the other.

MATERIALS AND METHODS: This is an objective study in which, patients with complaints of pain and instability of knee attending to the Department of Orthopaedics, Malla Reddy Hospital (MRH) attached to Malla Reddy Institute of Medical Sciences (MRIMS), Surarum, Hyderabad, were studied. More than 200 patients reported to the outpatient department between $1^{\text {st }}$ January 2012 and $31^{\text {st }}$ August 2012. Of which 50 patients underwent clinical examination, MRI and Arthroscopic evaluation.

The age group of these patients ranges from 11 years to 55 years. Out of 50 patients 41 were males and nine were females. History of the patient was taken. Patients were examined thoroughly. Tests used for menisci were joint line tenderness, McMurray's test, Apleys Grinding test. Applicable tests were used with respect to patient's complaints and history. Tests used for cruciate ligament assessment were anterior and posterior Drawer tests, Lachaman's test, Pivot shift test, Recurvatum external rotation tests, Quadriceps active test. Clinical diagnosis was made.

MRI was taken and films were read and MRI diagnosis was made. Patients were subjected to arthroscopy and surgical diagnosis was made. All the three results were correlated.

\section{STATISTICS AND ANALYSIS:}

1. Age distribution: 27 patients were between 11 and 30 years. There were 23 patients between 31 to 55 years.

2. Sex distribution: 41 patients were males and nine were females

3. Side distribution: Among the 50 patients 21 were right sided and 29 left sided.

4. Nature of injury: 40 patients had history of trauma and 10 patients had history of sports injury.

5. Diagnosed as per Clinical/MRI/ Arthroscopy:

All the 10 cases of isolated ACL injury and one case of only PCL injury, one case of both ACL \& PCL injury, there was equal correlation between Clinical, MRI, Arthroscopy (table 1).

In Meniscal injuries including only menisci $(n=5)+$ menisci + ACL $(n=26)$ there were 31 diagnosed on arthroscopy, 5 were misdiagnosed clinically but on arthroscopy had no tear (table 1). 


\section{ORIGINAL ARTICLE}

So sensitivity was $88 \%$ and specificity was $97 \%$ for meniscal injuries by clinical examination. Out of 50, MRI diagnosed 40 cases properly and two cases were not diagnosed and three cases were falsely diagnosed. So specificity was $93 \%$ and sensitivity was $93.5 \%$ for meniscal injuries by MRI examination (table 2) \& (fig 1).

In both meniscal and cruciate ligament injuries 32 cases were diagnosed by clinical examination, out of which Arthroscopically 26 were diagnosed and 28 were diagnosed by MRI (table 1). So specificity was $100 \%$ and sensitivity was $90 \%$ by clinical examination. So specificity was $98 \%$ and sensitivity was $93 \%$ for MRI (table 3) \& (fig 2) .

SUMMARY: Fifty cases were evaluated during $1^{\text {st }}$ January 2012 to $31^{\text {st }}$ August 2012 in Dept. of Orthopaedics, Malla Reddy Hospital/MRIMS, Hyderabad.

This study included the patients coming with complaints of pain \& instability in the knee. They were segregated clinically for meniscal and cruciate ligament injuries.

41 cases were males with left knee being predominant.

The youngest patient was eleven years and the oldest was fifty five years.

All the cases were evaluated clinically, MRI, and subjected to arthroscopy.

10 cases involved only ACL injuries which were diagnosed clinically, MRI and Arthroscopically.

Two PCL and bicruciate ligaments were diagnosed clinically, MRI and Arthroscopy. In both meniscal and cruciate ligament injuries 32 cases were diagnosed by clinical examination, out of which Arthroscopically 26 were diagnosed and 28 were diagnosed by MRI. In isolated meniscal injuries there were five diagnosed on arthroscopy, eight on clinical examination \& four on MRI. Five were misdiagnosed clinically but on arthroscopy had no tear.

CONCLUSION: From our study we concluded that,

1. For isolated ACL and PCL injuries Clinical, MRI and Arthroscopy are almost equal in diagnosing the condition.

2. For Meniscal injuries the sensitivity was $88 \%$ and specificity was $97 \%$ by clinical examination, sensitivity was $93.5 \%$, specificity was $93 \%$ by MRI, sensitivity was $100 \%$ and specificity was $100 \%$ for Arthroscopy.

3. In both Meniscal and Cruciate Ligament specificity was $100 \%$ and sensitivity was $90 \%$ by clinical examination, specificity was $98 \%$ and sensitivity was $93 \%$ for MRI, sensitivity was $100 \%$ and specificity was $100 \%$ for Arthroscopy.

4. Therefore Arthroscopy is best indicator of Cruciate Ligament as well as Meniscal injuries.

\section{BIBLIOGRAPHY:}

1. Campbell's Operative Orthopaedics, $11^{\text {th }}$ Ed. Chapter43;p 2416\&2417.

2. O'Connor J. Goodfellow J. Biden E: DL, Designing the human knee.pp52-64. In stokes IAF (Ed): Mechanical Factors and the skeleton. John Kibbey, London 1981.

3. Piaziali RL, Seering WP, Nagel DA, SChurman DJ: The function of the primary ligaments of the knee in anterior-posterior and medial-lateral motions. J. Biomech 13:777-784, 1980.

4. Tery GC, Target BE, Young MJ. Reliability of the clinical assessment in predicting the cause of internal derangement of the knee. Arthroscopy 1995; 11:568-576. 
5. Abdon P. Arnbojornsson AH, Egrund N, Lindstrand A, odenbring S, Pettersson H. lateral meniscal lesions in patients with clinically suspected medical lesions, Acta Orhop Scand 1989; 60:453-456.

6. Munk B. Madsen F, Lundorf E: Clinical MRI and Arthroscopic findings in knees. Journal of Arthoscopy 1998 March; 4(2):171-5.

7. Hughston JC: Acute knee injuries in athletes, Clin Orthop 23:114, 2962.

8. Gillies, Hamish, Seligson, David: Precision in the diagnosis of meniscal lesions: A Comparison of clinical evaluation, Arthrography, and Arthroscopy. JBJS 61A, 343-346, April 1979.

9. Edwin H.G, Oei, MSc, Jeroen J. Nikken MD, Antonio C.M. vertijinen, MSc Abida Z Ginai, MD, PhD and M.G.Myriam Hunink, MD, PhD: MR Imaging of the menisci and cruciate ligaments: A Systemic review Radiology 2003;226:873-848.

10. Radiology, Vol 164,445-448, Copyright @ 1987 by Radiological Society of North America.

11. De Smet AA, et al; AJR Am J Roentgenol. 1993 Jul; 161(1):101-7.

12. Riecher MA et al; Radiology, 1986 Jun; 159(3):753-7.

13. Van Dyck P. arch Orthop Trauma Surg. 2007 Nov; 127(9):849-54. Epub 2007 Apr.

14. Radiology 1995 Sep; 196(3):617-21.

15. Clinical, MRI, and Arthoscopic correlation in Meniscal and Anterior Cruciate Ligament injuries, F. Rayan, Sachin Bhonsle and Divyang D. Shukla: International Orthopaedics. 33(1):129-132. 2009 Feb.

16. Esmaili Jah Ali Akbar; Keyhani Sohrab; Zarfei Reza; Kalhor moghaddam Ali; Acta Orthopaedica Belgica. 71(2);189-196. 2005.

TABLE 1: Diagnosed as per Clinical/MRI/ Arthroscopy.

\begin{tabular}{|l|l|l|l|}
\hline DIAGNOSIS & CLINICAL & MRI & ARTHROSCOPY \\
\hline ONLY ACL & 10 & 10 & 10 \\
\hline ONLY MENISCI & 8 & 4 & 5 \\
\hline ONLY PCL & 2 & 1 & 1 \\
\hline MENISCI \& ACL & 32 & 28 & 26 \\
\hline ACL \& PCL & 3 & 1 & 1 \\
\hline
\end{tabular}

TABLE 2: Sensitivity and specificity for meniscal injuries.

\begin{tabular}{|l|l|l|l|}
\hline & CLINICAL & MRI & ARTHROSCOPY \\
\hline SENSITIVITY & 88 & 93.5 & 100 \\
\hline SPECIFICITY & 97 & 93 & 100 \\
\hline
\end{tabular}




\section{ORIGINAL ARTICLE}

FIGURE 1: Sensitivity and specificity for meniscal injuries.

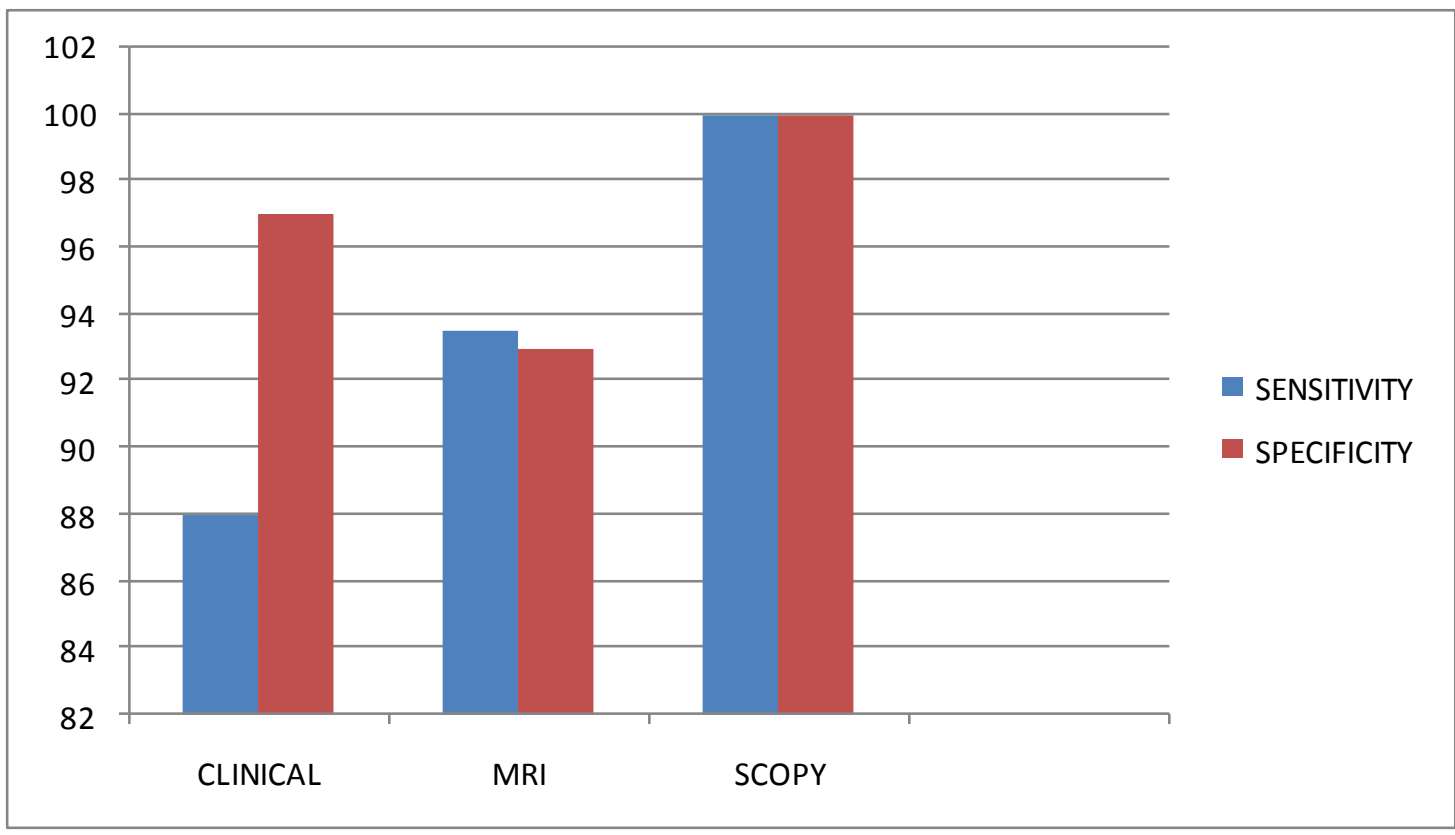

TABLE 3: Sensitivity and specificity for both meniscal and cruciate ligament injuries.

\begin{tabular}{|l|l|l|l|}
\hline & CLINICAL & MRI & ARTHOSCOPY \\
\hline SENSITIVITY & 90 & 93 & 100 \\
\hline SPECIFICITY & 100 & 98 & 100 \\
\hline
\end{tabular}

FIGURE 2: Sensitivity and specificity for both meniscal and cruciate ligament injuries.

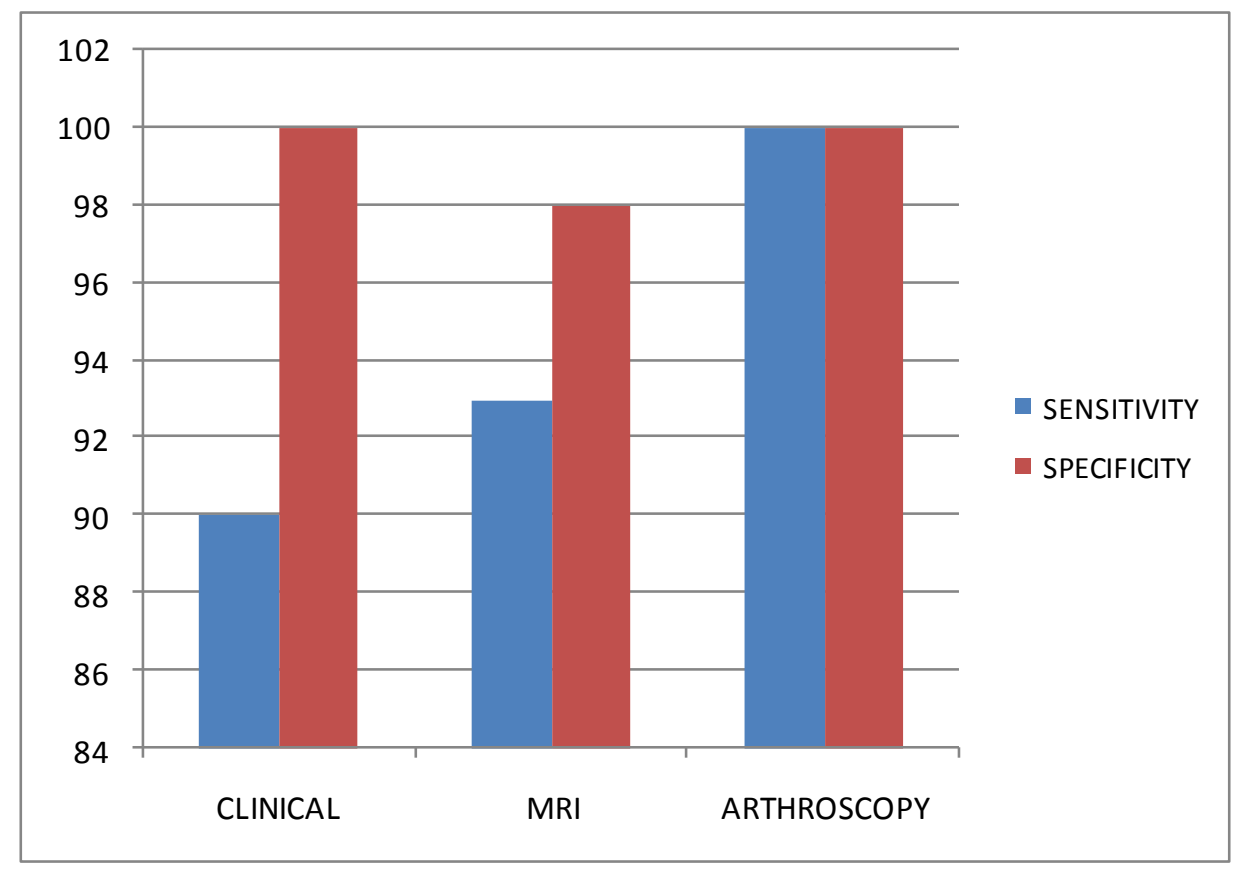

